\title{
Electrophysiological dissociation of retrieval orientation and retrieval effort
}

\author{
WILLIAM G. K. ROBB and MICHAEL D. RUGG \\ University College London, London, England
}

\begin{abstract}
The neural correlates of retrieval orientation - the differential processing of retrieval cues according to the form of the sought-for information-and retrieval effort were investigated in a factorial design. ERPs elicited by test words were recorded during four recognition memory tests. Orientation was manipulated by varying study material: The study phases preceding two of the tests employed pictures, whereas the study phases preceding the other two tests employed words. Effort was manipulated by varying difficulty, using a combination of the variables of length of study list and study-test interval. ERPs elicited by correctly classified new test words were sensitive to both the study material and, to a much lesser extent, the difficulty of manipulations. Whereas difficulty effects onset early and were shortlived, the effects of study material onset later, extended for several hundred milliseconds, and did not vary according to difficulty. It was concluded that retrieval orientation exerts a major influence on the processing of recognition memory test items.
\end{abstract}

The retrieval of an episodic memory requires an appropriate interaction between a retrieval cue and the encoded memory representation (Tulving, 1983). The importance of retrieval cues and the nature of their processing is emphasized in the principles of transfer appropriate processing (Morris, Bransford, \& Franks, 1977) and encoding specificity (Tulving \& Thomson, 1973). Broadly speaking, these principles propose that memory performance depends on the degree to which processing engaged at encoding is recapitulated at retrieval. These principles are supported by experiments in which processing at the time of study and the processing of retrieval cues in a subsequent test phase were independently manipulated (e.g., Roediger, Weldon, \& Challis, 1989).

The foregoing principles are based on evidence that direct manipulation of retrieval cue processing affects the probability of successful retrieval. There is some evidence, however (discussed below), that people are able to vary how they process retrieval cues even in the absence of specific test instructions or specially constructed cues. Such an ability would benefit memory by allowing the processing of a retrieval cue to be optimized in light of knowledge about the nature of the sought-for information. The aim of the present study was to strengthen the evidence for the existence of this ability.

According to Rugg and Wilding (2000), the ability to spontaneously vary the processing of a retrieval cue requires the ability to adopt different "retrieval orientations." In a re-

W.G.K.R. was supported by a Wellcome Prize Studentship.M.D.R. is supported by the Wellcome Trust and a cooperative grant from the U.K. Medical Research Council. We thank John Wixted and Tim Curran for comments on earlier versions of this paper. Correspondence should be addressed to M. D. Rugg. Institute of Cognitive Neuroscience, 17 Queen Square, London WC1N3AR, U.K. (e-mail: m.rugg@ucl.ac.uk). view of studies of episodic retrieval employing ERP (eventrelated potential) and functional neuroimaging methods, Rugg and Wilding proposed that a distinction should be drawn between processes that operate on a retrieval cue during an attempt to retrieve information from memory (preretrieval processes) and processes that operate on the products of a retrieval attempt (postretrieval processes). These latter processes include the maintenance of retrieved information and its evaluation in service of behavioral goals. They allow the operation of metamemorial strategies such as the "distinctiveness heuristic" (e.g., Schacter, Israel, \& Racine, 1999) and the "it-had-to-beyou" effect (Johnson, Raye, Foley, \& Foley, 1981).

Rugg and Wilding (2000) identified two classes of preretrieval processes that had been postulated on the basis of ERP and neuroimaging findings. One of these is retrieval orientation - the biasing of cue processing to meet the demands of a particular retrieval task. The second class of preretrieval processes is termed retrieval "effort." This term refers to the mobilization of processing resources in service of a retrieval attempt and is operationalized in terms of relative difficulty, the assumption being that the more difficult the retrieval task, the greater the effort expended (see, e.g., Buckner, Koutstaal, Schacter, Wagner, \& Rosen, 1998). Although nebulous, and of doubtful value from the perspective of a functional analysis of memory retrieval, the concept of retrieval effort serves as a useful reminder that many manipulations intended to vary the qualitative nature of the processes engaged during a retrieval task will also vary the difficulty of the task. This is important in the context of studies using measures of neural activity to index different forms of retrieval processing, since variations in difficulty per se may have neural correlates that could be confounded with, or even mistaken for, the correlates of different retrieval processes. 
Rugg and Wilding (2000) proposed that the neural correlates of retrieval orientation could be revealed by contrasting neural activity elicited by physically identical retrieval cues when these were used to probe memory for different kinds of information. They also proposed that these contrasts should be restricted to retrieval cues bearing no relation to studied items (e.g., new items in a recognition memory test), so as to avoid confounding pre- and postretrieval processing. They argued that cues corresponding to unstudied items would fully engage preretrieval processes, but unlike cues eliciting successful retrieval, they would engage postretrieval processes to only a limited degree.

In the present study we assessed the validity of the notion of retrieval orientation by asking whether retrieval cues are processed differently depending on the nature of the encoded information. This issue is well suited to investigation with physiological measures, when it reduces to the following questions: (1) Is neural activity elicited by a common class of retrieval cues sensitive to a putative manipulation of orientation?(2) Are these neural correlates of orientation, should they exist, distinct from the neural correlates of effort? As noted by Rugg and Wilding (2000), however, evidence addressing these questions is sparse because almost all potentially relevant studies have confounded either orientation and effort or pre- and postretrieval processing (by failing to separate neural activity elicited by cues eliciting successful vs. unsuccessful retrieval; e.g., Heil, Rösler, \& Hennighausen, 1996). Findings from two ERP studies in which these confounds were avoided suggest that different retrieval orientations are neurally dissociable (Johnson, Kounios, \& Nolde, 1997; Wilding, 1999; see also Ranganath \& Paller, 1999), but the findings do not speak to the question of whether the effects of orientation vary according to effort. In both studies the critical conditions involved ERPs elicited by items in tests of source memory. In Johnson et al. (1997), retrieval orientation was varied by a manipulation of study task, whereas in Wilding (1999), it was varied by the requirement to recover information about different attributes of study episodes. In each case, ERPs elicited by unstudied items varied with orientation, despite there being little difference in the difficulty of the retrieval tasks. There is evidence that ERPs may also be sensitive to retrieval orientation when the test task is yes/no recognition memory. Rugg, Allan, and Birch (2000) reported that ERPs elicited by new test words differed according to whether they were intermixed with "deeply" or "shallowly" encoded old words. Interpretation of this finding is difficult, however, because the encoding manipulation and test difficulty were confounded.

In the present study the ERP correlates of retrieval orientation were investigated by varying the form of the study material while holding constant the form of the retrieval cue. The study builds on previous findings in two ways. First, old/new recognition was employed rather than a test of source memory. Thus, the question whether previous findings extend to tasks where there is no explicit re- quirement to retrieve highly differentiated information is addressed. Second, a factorial design was employed in which task difficulty and study material were varied orthogonally. It was therefore possible to assess the extent to which any ERP correlate of retrieval orientation is independent of retrieval effort.

\section{METHOD}

\section{Participants}

Data are reported from 19 right-handed young adults (11 female). Data from a further 9 participants were rejected due to insufficient trials $(<16)$ in one or more critical experimental conditions, resulting from the combination of poor performance and too few artifactfree trials.

\section{Items}

Items were 390 color pictures of nameable objects and 390 words corresponding to the names of these objects; 288 pictures with unanimous or near-unanimous name agreement among pilot participants were selected as critical items, along with their corresponding names. The remaining 102 items of each type were employed as fillers. The 288 pictures were randomly allocated into eight lists of 36 items each, and their corresponding words were then allocated into a parallel set of eight lists. Test lists comprised words only, whereas study lists were made up of either pictures or words. For each study list containing pictures, the test list consisted of a random ordering of the names corresponding to those pictures along with 36 unstudied names. For study lists containing words, the test lists comprised the same words along with 36 new ones. Test lists were buffered with filler items at the beginning of the list and in the position immediately after the midlist pause (see below). Study lists were buffered with an initial filler item. In addition, for the "hard" study lists, additional filler items were included at randomly chosen locations to increase list length. On the basis of pilot studies, an extra 36 and 54 items were added to the word and picture lists, respectively, producing approximately equivalent performance decrements for each item type relative to performance in the "easy" conditions. Across lists every critical name served as an old and a new item in both easy and hard picture and word conditions.

\section{Procedure}

Study items were presented on a computer monitor for a duration of $1,500 \mathrm{msec}$, and were preceded for $500 \mathrm{msec}$ by a fixation character. Pictures (maximum visual angle $2.25^{\circ} \times 2.25^{\circ}$ ) were presented in color on a light gray background. Words were presented in white on black in lowercase with a maximum visual angle of $2.5^{\circ} \times$ $0.5^{\circ}$. For pictures, the study task was to judge whether the depicted item would in real life be bigger or smaller than a newspaper. For words, participants judged whether the named object would be more likely to be found indoors or outdoors. These tasks were chosen to encourage imagery-based encoding for pictures and a more "functional" form of encoding for words, the aim being to maximize differences between the memory representations formed for the two classes of study material. In each case, judgments were signaled by keypress with one or the other index finger.

Each test trial began with the display of a fixation character for $1,200 \mathrm{msec}$. Items were displayed $100 \mathrm{msec}$ after fixation offset for a duration of $500 \mathrm{msec}$, and were followed by a second fixation character that remained on the screen for $2,444 \mathrm{msec}$. The fixation character signaling the next trial appeared $200 \mathrm{msec}$ later. When pictures were the study items, test instructions were to judge whether or not each word was the name of a studied picture, signaling the judgment by making a left- or right-hand keypress. When words had been studied, participants judged whether each test word had been shown at study. For the two easy study-test blocks, the study test in- 
terval was $30 \mathrm{sec}$, which was filled by a backward counting task. For the two hard blocks, the interval was $5 \mathrm{~min}$. Each test list was interrupted by a brief rest break after presentation of half the items.

Participants practiced with short study and test lists prior to the first study task proper. Study material was blocked in such a way that either the two picture or the two word blocks were performed first, with the order alternated over participants. Every possible ordering of the hard and easy conditions was rotated across participants. The hand used to make old and new judgments remained constant across blocks, but was alternated across participants. Study and test lists were allocated to participants so that critical items rotated over the picture/word, hard/easy, and old/new manipulations.

\section{ERP Recording and Analysis}

EEG was recorded from 31 silver/silver chloride electrodes. Twenty-nine of these were embedded in an elasticated cap (montage as depicted in Figures 1 and 2) with the remaining electrodes placed on each mastoid process. Recordings were made with reference to the midfrontal electrode (Fz of the international 10-20 system) and subsequently rereferenced to linked mastoids. Vertical and horizontal EOGs were recorded from electrode pairs above and below the left eye and on the outer canthi. EEG and EOG were amplified with a bandwidth of $0.03-35 \mathrm{~Hz}$ (3-dB points) and digitized at $125 \mathrm{~Hz}$. The recording epochs began $104 \mathrm{msec}$ prior to stimulus onset for a duration of 2,048 $\mathrm{msec}$. A correction procedure was applied to minimize trials rejected due to blink artifact, using linear regression to estimate and correct the contribution of blink artifact to the EEG. Trials containing horizontal or vertical eye movements other than blinks were rejected, as were trials with A/D saturation or baseline drift exceeding $\pm 55 \mu \mathrm{V}$.

ERPs were formed for correctly classified test items from each condition. Participants were excluded if fewer than 16 trials were available to form ERPs from a condition. ERPs were digitally smoothed ( $3 \mathrm{~dB}$ down at $19.4 \mathrm{~Hz}$ ) and quantified by measurement of the mean amplitude (with respect to mean prestimulus baseline) of selected latency regions. Condition effects were assessed by analysis of variance (ANOVA) of data from selected electrode sites. Sphericity violation was corrected with the Geisser-Greenhouse procedure (Winer, 1971) and $F$ ratios are reported with corrected degrees of freedom.

\section{RESULTS}

\section{Behavior}

Recognition memory performance is summarized in Table 1. Accuracy and bias were measured by the parameters $\mathrm{Pr}$ (i.e., pHit-pFalse Alarm) and $\mathrm{Br}$ (Snodgrass \& Corwin, 1988). The means for Pr were .62, .79, .44, and .64 for the easy picture, easy word, hard picture, and hard word conditions, respectively. The corresponding values of $\mathrm{Br}$ were $.37, .41, .42$, and .38. ANOVA of Pr revealed main effects of difficulty $[F(1,18)=59.23, p<.001]$ and study material $[F(1,18)=39.23, p<.001]$, but no diffi- culty $\times$ material interaction $(F<1)$. ANOVA of $\mathrm{Br}$ revealed no significant effects.

ANOVA of reaction times (RTs) (factors of response [hit vs. correct rejection], difficulty, and study material) gave rise to main effects of response $[F(1,18)=28.21$, $p<.001]$, difficulty $[F(1,18)=9.54, p<.01]$, and material $[F(1,18)=78.152, p<.001]$, and to interactions between response and difficulty $[F(1,18)=5.46, p<.05]$ and response and material $[F(1,18)=12.13, p<.005]$. ANOVA of the correct rejection RTs alone revealed main effects of difficulty $[F(1,18)=11.68, p<.003]$ and material $[F(1,18)=66.84, p<.001]$, but no difficulty $\times$ material interaction $[F(1,18)=2.27]$.

\section{ERPs}

Grand average ERP waveforms from the three midline electrodes are shown in Figure 1 for correctly classified new items, collapsed over all factors apart from difficulty (left column) and study task (right column). Preliminary analyses of consecutive 100-msec latency intervals showed that difficulty effects were significant until $300 \mathrm{msec}$ poststimulus, whereas study material (but not difficulty) effects were present from around $300 \mathrm{msec}$ onward. These analyses also showed that the general pattern of the findings was little altered by selection of data from different recording sites. We report below the results of ANOVAs of mean amplitudes at midline electrodes between 0 and $300 \mathrm{msec}$, and 300- and 1,900-msec poststimulus, as these intervals capture the key aspects of the effects of the difficulty and material manipulations on the ERP data. In each case, factors were difficulty (easy vs. hard), study material (picture vs. word), and electrode site.

ANOVA of the $0-300 \mathrm{msec}$ interval revealed a significant interaction between difficulty and site $[F(1.3,24.0)=$ $6.30, p<.025]$, reflecting the tendency for waveforms to be more positive-going for the easy condition at the frontal site, but more positive-going for the hard condition at the central and parietal sites. By contrast, ANOVA of the 300-1,900 msec interval gave rise to a significant material effect $[F(1,18)=27.46, p<.001]$, but no effect for difficulty and no interactions involving difficulty and material $\left(F_{\mathrm{s}}<1\right)$. Collapsed across the three electrode sites, the mean difference between the word and picture conditions was $2.15 \mu \mathrm{V}$ for the easy tests and $2.87 \mu \mathrm{V}$ for the hard tests (see also Figure 1 insert).

As is evident in Figure 1, material effects on ERPs to new items extended over virtually the entire recording epoch post-300 msec. The question arises whether these

Table 1

Percent Correct in Each Experimental Condition and Associated Reaction Times (in Milliseconds)

\begin{tabular}{|c|c|c|c|c|c|c|c|c|c|c|c|c|c|c|c|}
\hline \multicolumn{8}{|c|}{ Easy } & \multicolumn{8}{|c|}{ Hard } \\
\hline \multicolumn{4}{|c|}{ Picture } & \multicolumn{4}{|c|}{ Word } & \multicolumn{4}{|c|}{ Picture } & \multicolumn{4}{|c|}{ Word } \\
\hline \multicolumn{2}{|c|}{ Old } & \multicolumn{2}{|c|}{ New } & \multicolumn{2}{|c|}{ Old } & \multicolumn{2}{|c|}{ New } & \multicolumn{2}{|c|}{ Old } & \multicolumn{2}{|c|}{ New } & \multicolumn{2}{|c|}{ Old } & \multicolumn{2}{|c|}{ New } \\
\hline RT & $\%$ & RT & $\%$ & $\mathrm{RT}$ & $\%$ & RT & $\%$ & RT & $\%$ & RT & $\%$ & RT & $\%$ & RT & $\%$ \\
\hline 1,060 & 76 & 1,190 & 86 & 983 & 88 & 1,008 & 92 & 1,150 & 63 & 1,303 & 77 & 986 & 77 & 1,053 & 87 \\
\hline
\end{tabular}



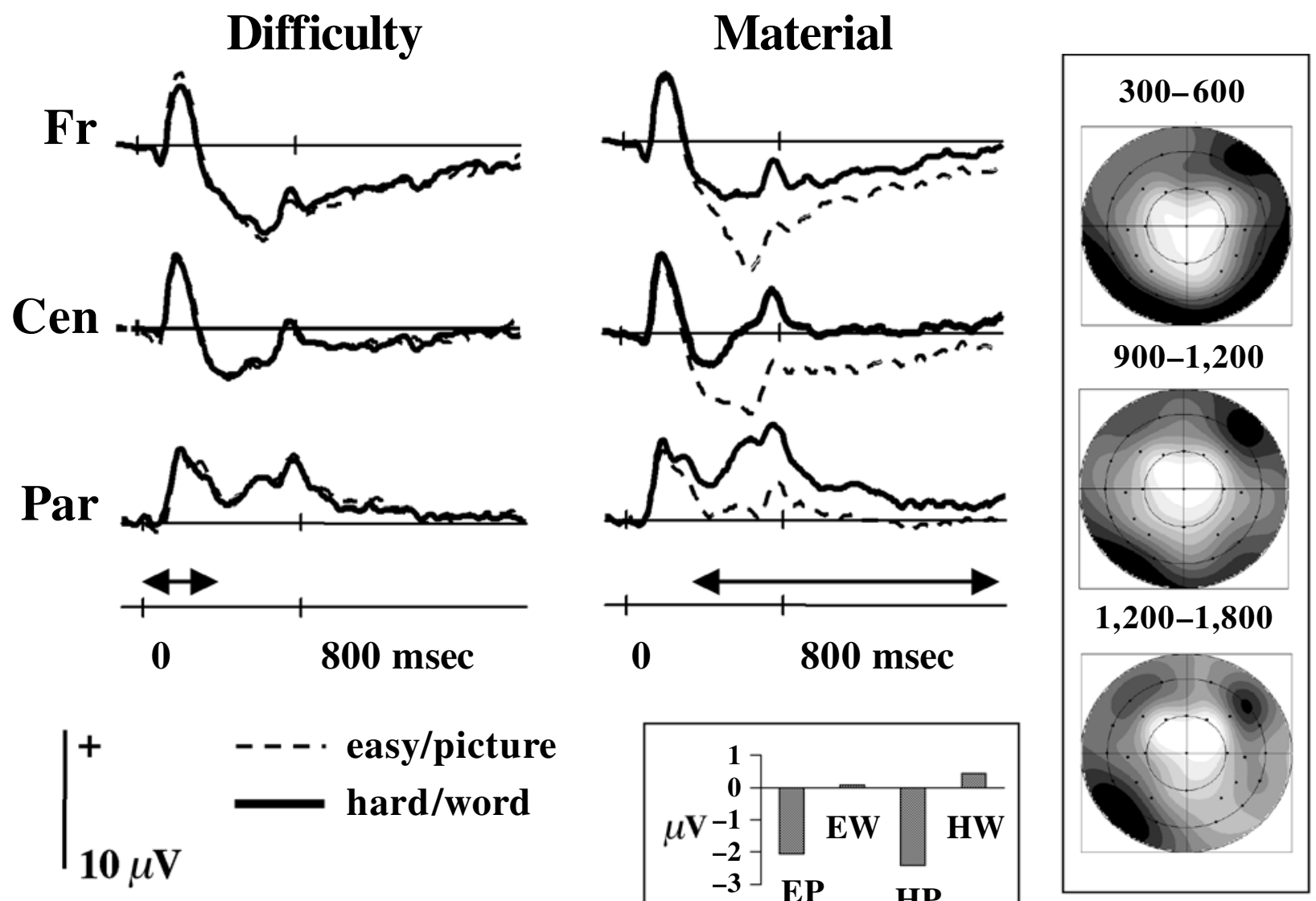

Figure 1. Main figure: grand average ERP waveforms $(n=19)$ from frontal (Fr), central (Cen), and parietal (Par) midline electrode sites. Left column: waveforms elicited by correctly classified new words in hard and easy conditions, collapsed across study material. Right column: waveforms elicited by correctly classified new test words following picture and word study tasks, collapsed across difficulty. Leftmost insert shows mean 300-1,900 msec amplitude across the three midline sites for ERPs in easy picture, easy word, hard picture, and hard word conditions, respectively. Right insert shows scalp distribution of the material effects (word minus picture) for the indicated latency intervals. Maps are normalized with respect to maxima and minima within each region; lighter shading indicates greater positivity.

effects reflect the activity of a single generator (or generator population) or multiple generators with differing time courses. This question is especially pertinent given that several topographically distinct ERP "components" (e.g., N400, P300, "slow wave"; Kutas \& Dale, 1997) are found in ERPs post-300 msec. To address the question, the scalp distributions of the material effects were compared across three latency regions, 300-600, 900-1,200, and 1,5001,800 msec (see Figure 1, right insert). This was done by ANOVA of the ERP difference scores (picture minus word) at every electrode site, employing the factors of latency region, difficulty, and site. The scores for each region were normalized as recommended by McCarthy and Wood (1985) to remove the confounding effects of interregion differences in mean amplitude. Evidence of timevarying changes in the configuration of the generators of the material effects would take the form of an interaction between latency region and electrode site, indicating that the profile of activity across the scalp differed with time.
The interaction was, however, nonsignificant $[F(2.2,47.9)=$ 2.04], as indeed were all other effects involving the material or difficulty factors (maximum $F=1.87$ ). Thus, in keeping with the impression given by Figure 1, there was no evidence that as time progressed different generator configurations, and hence different ERP components, contributed to the effects of material. ${ }^{1}$

For the reasons outlined in the introduction, the foregoing analyses were restricted to the waveforms elicited by new items. The effects of study material were also compared with the effects of retrieval success, the latter operationalized as the difference between ERPs to correctly classified old and new test items. These analyses were conducted on data from the midline parietal electrode site in the two easy conditions, as it was at this site and in these conditions that old/new effects were largest. Data from 3 participants for whom there were fewer than 16 trials in the ERPs elicited by old items were excluded from these analyses. The relevant waveforms are shown 
in Figure 2, where it can be seen that, as described many times previously, old items elicited more positive-going waveforms than did new items (for a review, see Friedman $\&$ Johnson, 2000). The old/new effects appear to onset later than the effects of study material. The onset latency of the effects was estimated by ANOVA (factors of material [picture vs. word], and item type [old vs. new]) of successive 100-msec intervals of these waveforms. Effects of study material first became reliable in the $200-300 \mathrm{msec}$ interval $[F(1,15)=6.01, p<.05$, when $F<1$ for old vs. new], whereas the old/new effects did not achieve significance until the 400-500 msec interval $[F(1,15)=11.81$, $p<.005]$. In neither of these intervals, nor in the intervening one $[F(1,15)=6.91, p<.025$, for the material effect), was there an interaction involving the factors of material and item type $\left(F_{\mathrm{S}}<1\right)$.

\section{DISCUSSION}

Difficulty and study material had additive effects on both recognition accuracy and correct rejection RTs, and had no significant effect on response bias. Crucially, performance differences due to difficulty were as large as the differences due to study material. Thus, if the effects on ERPs of study material merely reflected the greater effort required to retrieve pictorial rather than verbal information, equivalent effects should have been apparent in contrasts of ERPs according to difficulty. There was, however, no overlap between the effects of difficulty and material. The behavioral findings further indicate that cognitive operations associated with maintaining different response criteria were not responsible for the observed ERP effects (see Ranganath \& Paller, 1999; Rugg et al.,
2000). The effects of study material on performance are consistent with the transfer appropriate processing principle (see the introduction). Evidently, whatever the differences in retrieval cue processing engendered by the requirement to retrieve pictures versus words (see below), these did not compensate entirely for the detrimental effects of incompatible study-test formats. ${ }^{2}$

The effects on ERPs of the study material manipulation were striking: From around 300 msec poststimulus ERPs elicited by new items were more negative-going when these items were employed to probe memory for pictures rather than words. For the reasons already noted, this effect cannot be attributed to differences in difficulty between the two kinds of retrieval task. Furthermore, since the effect was obtained with correctly classified new items, it cannot be a reflection of processing associated with, or contingent upon, positive recognition judgments (so-called retrieval success effects). The findings are therefore consistent with the proposal that participants adopted different retrieval orientations (Rugg \& Wilding, 2000) when attempting to retrieve pictures as opposed to words.

Retrieval orientation is assumed to affect preretrieval processing and to influence the way in which cues are employed to probe memory (Rugg \& Wilding, 2000). The finding that the onset of study material effects preceded the onset of old/new effects is consistent with this assumption in that it suggests that differential cue processing can begin before retrieval of the study items. Thus, early on, at least, the material effects are unlikely to reflect differences in postretrieval processing. The early onset of these effects does not, however, rule out the possibility that, at a later time, the effects reflected other as-

\section{Material}

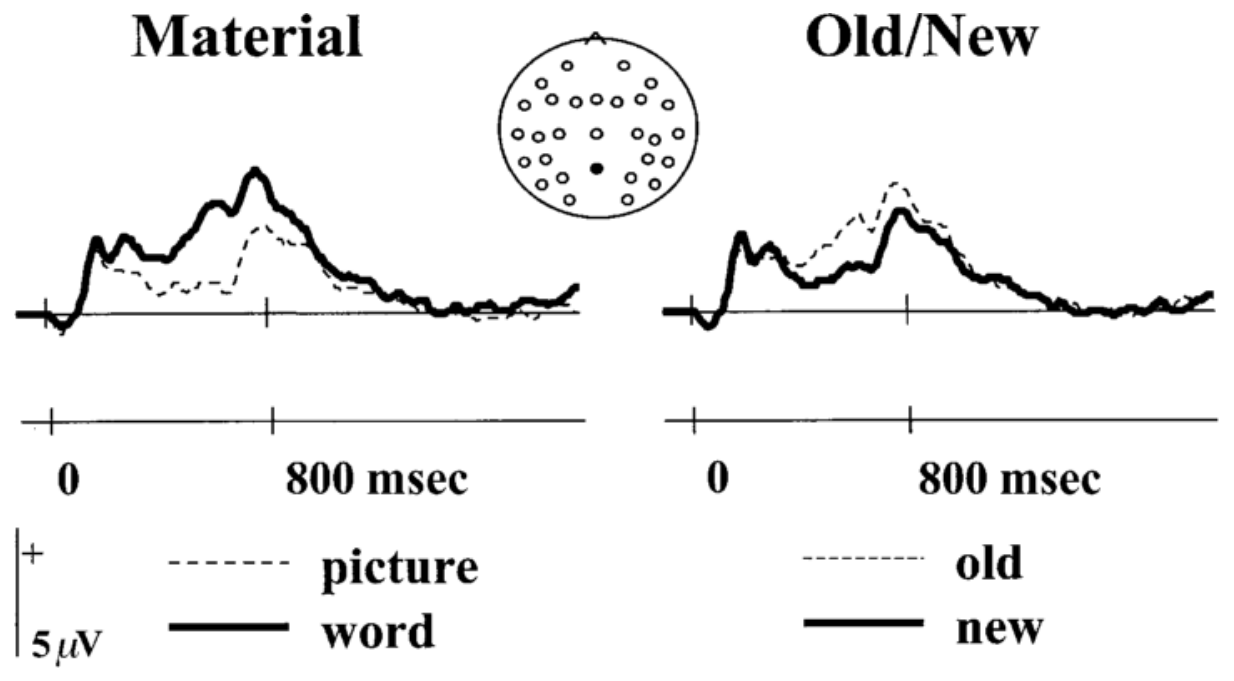

Figure 2. Grand average ERP waveforms $(N=16)$ from the midparietal electrode site in the easy conditions. Left: waveforms elicited by correctly classified test words following picture and word study tasks, collapsed across words corresponding to old and new items. Right: waveforms elicited by correctly classified old and new test words, collapsed across study material. 
pects of retrieval processing also. For example, the processes engaged to monitor and evaluate the outcome of retrieval attempts (e.g., Burgess \& Shallice, 1996; Henson, Rugg, Shallice, \& Dolan, 2000) might have differed according to the form of the information probed for, and these differences might have contributed to the observed ERP effects. However, inasmuch as these processes would be expected to have distinct neural signatures, the finding that the scalp distribution of the material effects remained constant with time is inconsistent with this possibility.

If the foregoing material effect does indeed uniformly reflect preretrieval processing, why is its time course so extended (lasting until about $1,800 \mathrm{msec}$ ), given that successful retrieval apparently began much sooner? There are two possible answers to this question, which are not mutually exclusive. First, the temporally extended material effect illustrated in Figure 1 was elicited by correctly classified new items, items for which preretrieval processing did not culminate in successful retrieval of a study episode. The latency at which successful retrieval began offers no guide to how long a retrieval cue would continue to be employed to probe memory in the absence of retrieval success. Second, the effect illustrated in Figure 1 represents the difference between waveforms averaged across numerous test trials. Thus, it can confidently be concluded that the differential processing reflected by the effect was, on at least some trials, initiated no later than the onset time of the effect. It can also be concluded that, on at least some trials, differential processing continued until the offset of the effect. But it cannot be concluded that the processes in question were active for the whole of the interval spanned by these times; to the degree that there was significant across-trial variation in the onset of these processes, their duration might have been considerably shorter.

At the functional level, the significance of the present findings is twofold. First, they support the proposal (see introduction) that people can vary how they process a recognition memory test item so as to optimize its effectiveness as a retrieval cue. We assume that the basis of this effect is the knowledge that retrieval is facilitated when overlap between cue and target processing is high, as demonstrated experimentally many times and embodied in theoretical principles such as transfer appropriate processing and encoding specificity (see the introduction). In the present case, for example, participants may have attempted to generate images of the referents of test words when trying to retrieve pictures, while relying on lexi$\mathrm{cal} / \mathrm{semantic}$ processing when retrieving words. Whether the knowledge underlying the adoption of different retrieval orientations is available explicitly, and contributes to a consciously maintained retrieval strategy, or whether instead it exercises only an implicit influence on cue processing, is an interesting question for the future.

One implication of this discussion is that when a common class of retrieval cues is employed to probe memory for materials encoded under different conditions, it may be necessary to consider the extent to which differences in retrieval performance are a consequence of different re- trieval orientations. For example, Dodson and Schacter (2001; see also Schacter et al., 1999) reported lower false alarm rates to semantic associates of study words when the study task involved reading words out loud than when words were read silently and heard passively. This finding was present, however, only when the study task was a blocked, between-subjects variable. Equivalent false alarm rates were observed for associates of the two classes of study word when task was varied randomly within-subjects. Dodson and Schacter interpreted their findings as evidence for the employment of a postretrieval "distinctiveness heuristic." According to the framework put forward here, another possibility is that the orientation adopted to retrieve "said" words was more effective in preventing "retrieval" of unstudied semantic associates than was the orientation adopted when retrieving "heard" words. Since the adoption of a consistent retrieval orientation would have been possible only when study task was a blocked variable (as was the case, of course, in the present experiment), this account, like Dodson and Schacter's, is consistent with the failure to find differential false alarm rates when task was randomized.

A second significant point about these findings is that they demonstrate the promise of this experimental approach for the study of retrieval cue processing. In the present case, the encoding manipulation was chosen to maximize the likelihood of detecting a difference due to retrieval orientation, and as a result it might be argued that the findings are not especially surprising. More subtle encoding manipulations will, however, allow investigation of the limits to the ability to adjust cue processing in response to changes in the nature of the encoded information. It should also be possible to establish whether this ability varies across different populations (e.g., young vs. older people) and the extent to which it is under voluntary control.

In marked contrast to the effects of study material discussed above, the effects on ERPs of difficulty were small and short-lived. They were also of sufficiently modest statistical significance to warrant replication before discussion of their possible functional significance. It remains to be established whether other ways of manipulating difficulty have more profound effects on ERPs. The key point for present purposes, however, is that the effects of the difficulty manipulation on behavior were equivalent to those of study material. As already noted, this means that the material effects on ERPs cannot be reduced to difficulty effects.

\section{REFERENCES}

Buckner, R. L., Koutstaal, W., Schacter, D. L., Wagner, A. D., \& Rosen, B. R. (1998). Functional-anatomic study of episodic retrieval using f MRI, I. Retrieval effort versus retrieval success. NeuroImage, 7, 151-162.

Burgess, P. W., \& Shallice, T. (1996). Confabulation and the control of recollection. Memory, 4, 359-411.

Curran, T. (2000). Brain potentials of recollection and familiarity. Memory \& Cognition, 28, 923-938.

Dodson, C. S., \& Schacter, D. L. (2001). "If I had said it I would have 
remembered it": Reducing false memories with a distinctiveness heuristic. Psychonomic Bulletin \& Review, 8, 155-161.

Friedman, D., \& JoHnson, R. (2000). Event-related potential (ERP) studies of memory encoding and retrieval: A selective review. $\mathrm{Mi}$ croscopy Research \& Technique, 51, 6-28.

Heil, M., Rösler, F., \& Hennighausen, E. (1996). Topographically distinct cortical activation in episodic long-term memory: The retrieval of spatial versus verbal information. Memory \& Cognition, 24, 777-795.

Henson, R. N. A., Rugg, M. D., Shallice, T., \& Dolan, R. J. (2000). Confidence in recognition memory for words: Dissociating right prefrontal roles in episodic retrieval. Journal of Cognitive Neuroscience, 12, 913-923.

Johnson, M. K., Kounios, J., \& Nolde, S. F. (1997). Electrophysiological brain activity and memory source monitoring. NeuroReport, $\mathbf{8}$, 1317-1320.

Johnson, M. K., Raye, C. L., Foley, H. J., \& Foley, M. A. (1981). Cognitive operations and decision bias in reality monitoring. American Journal of Psychology, 94, 37-64.

Kutas, M., \& Dale, A. (1997). Electrical and magnetic readings of mental functions. In M. D. Rugg (Ed.), Cognitive neuroscience (pp. 197-241). Hove, U.K.: Psychology Press.

McCarthy, G., \& Wood, C. C. (1985). Scalp distributions of eventrelated potentials: An ambiguity associated with analysis of variance models. Electroencephalography \& Clinical Neurophysiology, 62, 203-208.

Mintzer, M. Z, \& Snodgrass, J. G. (1999). The picture superiority effect: Support for the distinctiveness model. American Journal of Psychology, 1, 113-146.

Morris, C. D., Bransford, J. D., \& Franks, J. J. (1977). Levels of processing versus transfer appropriate processing. Journal of Verbal Learning \& Verbal Behavior, 16, 519-533.

Ranganath, C., \& Paller, K. A. (1999). Frontal brain potentials during recognition are modulated by requirements to retrieve perceptual detail. Neuron, 22, 605-613.

Roediger, H. L., III, Weldon, M. S., \& Challis, B. H. (1989). Explaining dissociations between implicit and explicit measures of retention: A processing account. In H. L. Roediger, III, \& F. I. M. Craik (Eds.), Varieties of memory and consciousness (pp. 3-41). Hillsdale, NJ: Erlbaum.

Rugg, M. D., Allan, K., \& BirCh, C. S. (2000). Electrophysiologicalevidence for the modulation of retrieval orientation by depth of study processing. Journal of Cognitive Neuroscience, 12, 664-678.

Rugg, M. D., Mark, R. E., Walla, P., Schloerscheidt, A. M., BIRCH, C. S., \& Allan, K. (1998). Dissociation of the neural correlates of implicit and explicit memory. Nature, 392, 595-598.
RugG, M. D., \& Wilding, E. L. (2000). Retrieval processing and episodic memory. Trends in Cognitive Sciences, 4, 108-115.

Schacter, D. L., Israel, L., \& Racine, C. (1999). Suppressing false recognition in younger and older adults: The distinctiveness heuristic. Journal of Memory \& Language, 40, 1-24.

Snodgrass, J. G., \& Corwin, J. (1988). Pragmatics of measuring recognition memory-Applications to dementia and amnesia. Journal of Experimental Psychology: General, 117, 134-150.

Stenberg, G., Radeborg, K., \& Hedman, L. R. (1995). The picture superiority effect in a cross-modality recognition task. Memory \& Cognition, 23, 425-441.

Tulving, E. (1983). Elements of episodic memory. Oxford: Oxford University Press.

Tulving, E., \& Thomson, D. M. (1973). Encoding specificity and retrieval processes in episodic memory. Psychological Review, 80, 353373.

WILDING, E. L. (1999). Separating retrieval strategies from retrieval success: An event-related potential study of source memory. Neuropsychologia, 37, 441-454.

WINER, B. J. (1971). Statistical principles in experimental design. New York: McGraw-Hill.

\section{NOTES}

1. The latency intervals included in the analyses of scalp topography were selected so as to sample the topography of the material effect across its entire extent in an unbiased fashion. An additional contrast between the topographies of this effect during the 300-500 msec and the $500-800 \mathrm{msec}$ latency regions, intervals previously found to contain dissociable, retrieval-related ERP components (Curran, 2000; Rugg et al., 1998), was also nonsignificant $(F<1)$.

2 . The finding of higher performance when words rather than pictures were the study material represents a reversal of the well-known "picture superiority effect." It should be noted, however, that (1) the effect is much diminished and sometimes reversed when, as here, recognition test items are words (Mintzer \& Snodgrass, 1999; Stenberg, Radeborg, \& Hedman, 1995); and (2) to maximize the difference between their respective memory representations, pictures and words were encoded using different study tasks, which may have differed in effectiveness.

(Manuscript received December 28, 2001; revision accepted for publication August 29, 2001.) 